\title{
Monitoring of Depth of Anesthesia Using Entropy Monitor in Off-pump Coronary Artery Bypass Graft Surgery: An Observational Study
}

\author{
Masood Ahmed Chandsha ${ }^{1}$, Naina Dalvi ${ }^{2}$
}

\begin{abstract}
Aims and objectives: To monitor the depth of anesthesia using entropy monitor during off-pump coronary bypass graft surgery and to assess memories or recall of intraoperative awareness using Modified Version of Brice Questionnaire after 48 hours of surgery.

Materials and methods: It is a cross-sectional observational study. After approval by the institutional ethics committee, the study was conducted in 100 patients posted for cardiac surgery involving off-pump coronary bypass surgery fulfilling the inclusion criteria. After written valid consent, general anesthesia was given as per the protocol. Entropy indices are calculated and displayed in real-time. The indices were manually recorded at various cardiac events. Controlled mechanical ventilation was continued in the ICU. Tracheal extubation was performed when hemodynamic and respiratory parameters remained stable. All patients were interviewed 48 hours after surgery about any memories or recall of intraoperative awareness using the modified Brice questionnaire.

Results: In our study, the hemodynamic parameters like pulse rate, systolic, and diastolic blood pressure were well maintained. Three patients reported awareness during the procedure. One of them had vivid dreams which the patient was not able to recall the nature of the dream and the other two patients who had woken up during the procedure heard some noises during the surgery. None of the patients reported being in pain when awake.

Conclusion: An entropy monitor might aid off-pump coronary artery bypass graft surgery (OPCABG) in preventing intraoperative awareness and monitoring the depth of anesthesia. It also aids in reducing anesthetic dosage and hence avoids untoward side effects of anesthesia.

Clinical significance: Our study suggested that the incidence of awareness is high during the OPCABG, hence modification in anesthesia technique is needed at present.

Keywords: Anesthesia awareness, Depth of anesthesia, Entropy, General anesthesia, Off-pump CABG, Recall.

Research and Innovation in Anesthesia (2021): 10.5005/jp-journals-10049-0094
\end{abstract}

\section{INTRODUCTION}

As believed, anesthesia and lack of consciousness are not interchangeable. There have been several instances, where patients remember different events during an operative procedure. And can present with neurosis and psychological disorders, which are referred to as "invisible scars of the surgery".

The activity survey data by the Royal College of Anesthetists in collaboration with the Association of Anesthetists of Great Britain and Ireland have studied the incidence of awareness in cardiac and thoracic surgery and it was found to be as high as 1 in 10,000 and 1 in 7,000, respectively. Surprisingly, both of these are higher than the average study rate. ${ }^{1}$

Nowadays, off-pump coronary artery bypass graft surgery (OPCABG) is preferred over traditional on-pump CABG as there is decreased postoperative morbidity seen with OPCABG and the cost of surgery is also lesser.

The maintenance of hemodynamic stability during accessing of coronary artery and the management of myocardial ischemia during grafting may necessitate lighter planes of anesthesia and subsequent awareness.

The entropy monitoring is used to measure the depth of anesthesia. The deeper the depth of anesthesia, the more regular is the EEG. This regularity is deduced by the entropy to estimate the depth of anesthesia.
${ }^{1}$ Department of Critical Care Medicine, Apollo Hospitals, Navi Mumbai, Maharashtra, India

${ }^{2}$ Department of Anaesthesiology, HBT Medical College and Dr RN Cooper Municipal Hospital, Mumbai, Maharashtra, India

Corresponding Author: Masood Ahmed Chandsha, Department of Critical Care Medicine, Apollo Hospitals, Navi Mumbai, Maharashtra, India, Phone: +91 9768615607, e-mail: shirinchandsha@gmail.com

How to cite this article: Chandsha MA, Dalvi N. Monitoring of Depth of Anesthesia Using Entropy Monitor in Off-pump Coronary Artery Bypass Graft Surgery: An Observational Study. Res Inno in Anesth 2021;6(1):11-16.

Source of support: Nil

Conflict of interest: None

\section{Materials and Methods}

This is a prospective cross-sectional observational study. Approval was taken from the Scientific Research Society and Ethics Committee. One hundred patients undergoing OPCABG who gave consent to participate in the study were selected. Patients of any gender between the ages of 18 and 60 were included in the study.

Patients with a background of neurological maladies, like epilepsy and thromboembolic events, and with a record of head injury were kept out from the study. 
The study was performed in Cardio-Vascular Thoracic Surgery (CVTS) OT of our hospital. Informed written consent was taken from the patients who met the inclusion criteria after briefing them about the study. Sufficient starvation was ensured on the day of surgery. The patient's valid written consent was obtained to monitor entropy values during the procedure. After taking the patient on the surgical table, a multipara monitor (GE DatexOhmeda) with cardioscope, pulse oximeter, cardio scope, and non-invasive blood pressure monitor was connected. The patient's blood pressure, heart rate, and oxygen saturation were checked and noted. Intravenous access was secured on the dorsum of the left hand using an 18/20G indwelling cannula. Routine general anesthesia as per the standard practice was given to all the participants in the study. Premedication was given in the form of injection fentanyl $2 \mu \mathrm{g} / \mathrm{kg}$ and Phenergan $0.5 \mathrm{mg} / \mathrm{kg}$ IV. Patients were given $100 \%$ oxygen for 3 minutes for preoxygenation using an anesthesia breathing system with a circle absorber and end-tidal carbon dioxide. Induction of anesthesia was done with injection midazolam $0.02 \mathrm{mg} / \mathrm{kg}+$ fentanyl 4 to $5 \mu \mathrm{g} / \mathrm{kg}$ + etomidate till loss of consciousness. Injection rocuronium $1 \mathrm{mg} /$ $\mathrm{kg}$ was given for neuromuscular blockade. Patients were ventilated with $\mathrm{O}_{2}$ and $\mathrm{N}_{2} \mathrm{O}$ for $2 \frac{1}{2}$ minutes followed by $100 \% \mathrm{O}_{2}$ for 30 seconds before intubation.

Under the direct laryngoscopic vision, oral intubation was done with an endotracheal tube, a polyvinyl chloride low pressure high volume cuffed tubes (Portex Inc.). All patients were mechanically ventilated using a circle absorber system and ventilator. Intraoperatively, anesthesia was maintained with oxygen, air, isoflurane along with dexmedetomidine infusion $(0.3-0.7 \mu \mathrm{g} / \mathrm{kg} /$ hour) and rocuronium infusion ( $0.45-0.6 \mathrm{mg} / \mathrm{kg} / \mathrm{hour}$ ). Nasogastric tube no 14 was inserted in all patients.

Entropy indices are calculated and displayed continuously. The indices will be manually recorded at various cardiac events immediate post-induction, skin incision, sternotomy, various cardiac positions during graft placement, at protamine injection, closure of sternal wire, skin closure. The safe limits for adequate anesthesia are ranging between 40 and 60 for both state entropy (SE) and response entropy (RE). Burst suppression ratio (BSR) was monitored only when the patient was too deep. Following completion of the surgery, the patient was not reversed and was not extubated. And was shifted to the cardiac recovery room for observation as per the routine protocol.
In every participant, variables like heart rate, blood pressure, $\mathrm{EtCO}_{2}$, temperature, and entropy values, were noted before and after induction. Heart rate, blood pressure, RE, SE, EtCO ${ }_{2}$, and temperature were noted at a fixed time interval and every step of the OPCABG procedure.

Every participant has interviewed 48 hours post-surgery for any remembrance or memory of intraoperative awareness using a modified Brice questionnaire.

Questions used to elicit frequency of recall:

- Before the procedure, what was the last thing you recollect??

- What is the first thing that comes to mind when you woke up?

- Did you dream when you were asleep during the procedure?

- Were you put to sleep gently?

- Did you face any difficulties going to sleep?

\section{Statistical Analysis, Observations, and Results}

SPSS 15.0 and SAS 9.2 software were used for devising tables of frequency distribution and percentages. Descriptive and inferential statistical analysis has been done. Results on continuous measurements are presented on mean \pm SD (Min-Max) and results on categorical measurements are presented in number (\%). The Student's $t$-test has been used to find the significance of study parameters on a continuous scale within each group. The associations between two variables were determined by the Pearson Chi-square test. For all statistical comparisons in this study, a $p$ value $<0.05$ was significant.

\section{Results}

The age of all 100 patients was between 41 years and 70 years with a mean and SD of $57.03 \pm 6.10$ years. There were 62 males and 38 females among the 100 participants. The mean and SD weight of the participants was $74.00 \pm 9.98 \mathrm{~kg}$ ranging from 50 to $90 \mathrm{~kg}$. The mean and SD height of the participants was $165.78 \pm 7.27 \mathrm{~cm}$ ranging from 146 to $180 \mathrm{~cm}$ (Fig. 1).

In this study, at immediate post-induction, the mean pulse rate was $86.11 \pm 10.70 /$ minute. At skin incision $84.60 \pm 9.86 /$ minute which significantly decreased with the progression of surgery and during various coronary artery bypass grafts with the least being for LCX

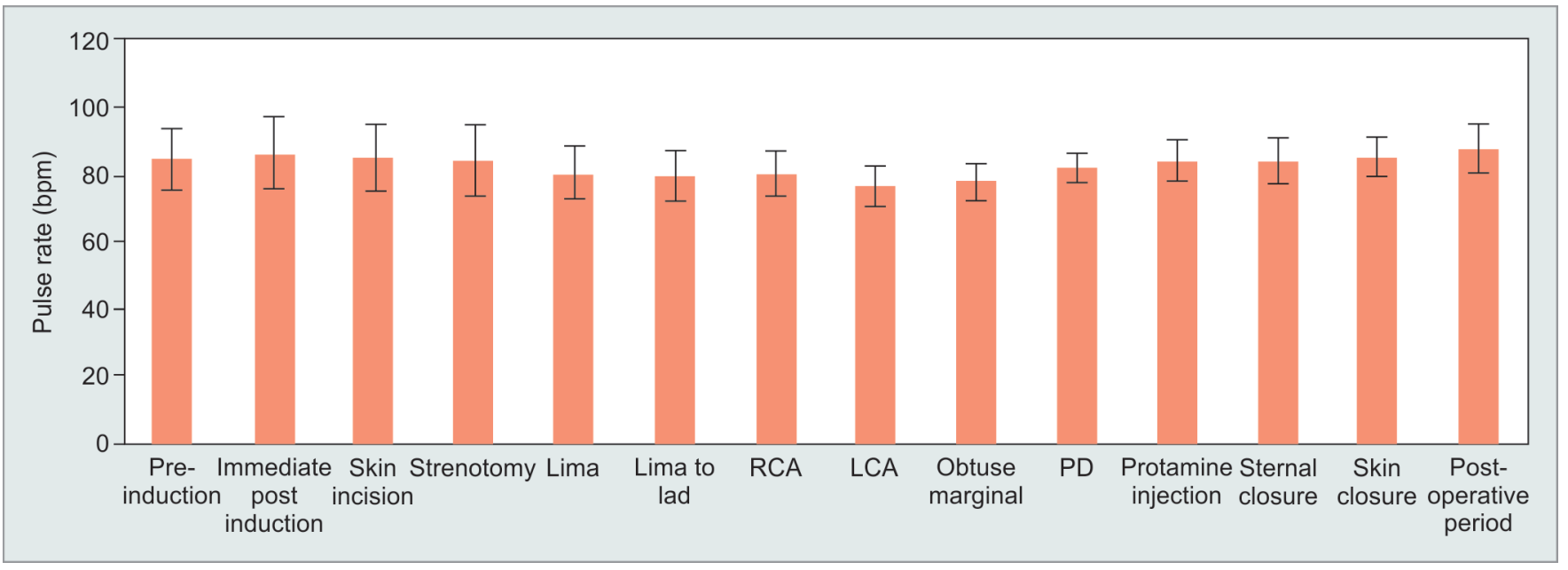

Fig. 1: Mean pulse rate during the procedure 


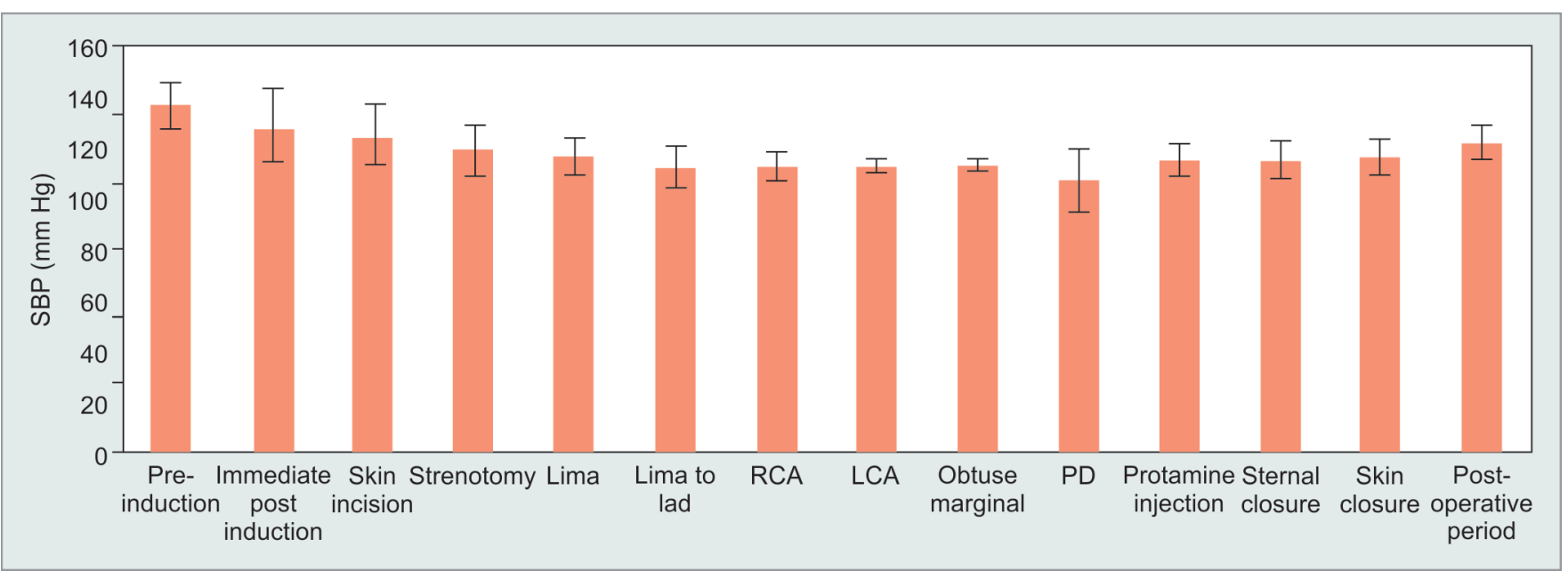

Fig. 2: Mean systolic blood pressure during the procedure

Table 1: Change in mean response entropy (RE) and mean state entropy (SE) from induction

\begin{tabular}{|c|c|c|c|c|c|c|}
\hline \multirow[b]{2}{*}{ Time } & \multicolumn{3}{|c|}{$R E$} & \multicolumn{3}{|c|}{ SE } \\
\hline & Mean $\pm S D$ & Difference & $p$ value & Mean $\pm S D$ & Difference & $p$ value \\
\hline Pre-induction & $94.66 \pm 2.44$ & - & - & $89.94 \pm 0.99$ & - & - \\
\hline Immediate post-induction & $53.93 \pm 10.56$ & 40.730 & - & $53.48 \pm 10.25$ & 36.460 & - \\
\hline Skin incision & $53.12 \pm 9.45$ & 41.540 & 0.087 & $51.46 \pm 9.46$ & 38.480 & $<0.001$ \\
\hline Sternotomy & $52.24 \pm 8.80$ & 42.420 & 0.051 & $50.62 \pm 8.47$ & 39.320 & 0.002 \\
\hline LIMA & $49.88 \pm 8.12$ & 44.780 & $<0.001$ & $49.02 \pm 7.88$ & 40.920 & $<0.001$ \\
\hline Lima to lad & $49.92 \pm 7.86$ & 44.740 & $<0.001$ & $49.28 \pm 8.10$ & 40.660 & $<0.001$ \\
\hline RCA & $50.15 \pm 8.12$ & 44.688 & $<0.001$ & $49.55 \pm 8.06$ & 40.463 & $<0.001$ \\
\hline LCX & $50.07 \pm 7.01$ & 44.964 & 0.010 & $48.93 \pm 7.81$ & 41.071 & 0.003 \\
\hline Obtuse marginal & $48.10 \pm 7.08$ & 46.571 & 0.026 & $48.10 \pm 7.08$ & 46.571 & 0.026 \\
\hline PD & $51.91 \pm 7.66$ & 42.545 & 0.772 & $51.18 \pm 7.07$ & 38.909 & 0.972 \\
\hline Protamine injection & $55.20 \pm 8.86$ & 39.460 & 0.299 & $54.64 \pm 9.12$ & 35.300 & 0.346 \\
\hline Sternal closure & $55.31 \pm 7.50$ & 39.350 & 0.158 & $54.09 \pm 7.53$ & 35.850 & 0.523 \\
\hline Skin closure & $56.51 \pm 8.34$ & 38.150 & 0.012 & $55.37 \pm 8.44$ & 34.570 & 0.041 \\
\hline
\end{tabular}

$76.32 \pm 5.98 /$ minute and obtuse marginal $77.52 \pm 5.51 /$ minute. The mean pulse rate increased during the end of the surgery which significantly increased at protamine injection $83.84 \pm 6.21 /$ minute and at the postoperative period mean pulse rate was $87.52 \pm 7.13$ / minute. The mean pulse rate during different cardiac positions was compared with the mean pulse rate during the pre-induction time and the difference was found statistically significant during left internal mammary artery to left anterior descending artery grafting, right coronary artery grafting, left circumflex artery, and obtuse marginal artery grafting (Fig. 2).

As per this study, at immediate post-induction, mean systolic pressure was $128.38 \pm 14.24 \mathrm{~mm} \mathrm{Hg}$. After sternotomy, mean pressure was $119.54 \pm 10.19 \mathrm{~mm} \mathrm{Hg}$ which significantly decreases till skin closure. At immediate post-induction, the mean diastolic pressure was $80.62 \pm 11.03 \mathrm{~mm} \mathrm{Hg}$. After sternotomy, mean pressure was $73.68 \pm 8.34 \mathrm{~mm} \mathrm{Hg}$ which significantly decreases till skin closure (Table 1).

In our study, the RE in awake patients was a mean of $94.66 \pm$ 2.44 , immediate post-induction the RE was $53.93 \pm 10.56$, which gradually decreased during various coronary artery bypass grafting (CABG), the RE started to rise gradually during protamine injection and toward skin closure, it was $56.51 \pm 8.34$. Similarly, the SE in awake
Table 2: 48 hours post-surgery patient assessment by modified Brice questionnaire

\begin{tabular}{llr}
\hline Recall & No. of patients & $\%$ \\
\hline Nil & 97 & 97.0 \\
Heard voices & 2 & 2.0 \\
Vivid dreams & 1 & 1.0 \\
Total & 100 & 100.0 \\
\hline
\end{tabular}

patients was a mean of $89.94 \pm 0.99$, immediate post-induction the SE was $53.48 \pm 10.25$, which gradually decreased during various $C A B G$, the $S E$ started to rise gradually during protamine injection and toward skin closure, it was $55.37 \pm 8.44$ (Table 2).

All patients were questioned after 48 hours in the postoperative period for awareness during surgery using a modified Brice questionnaire. Three patients reported awareness during the procedure. One of them had vivid dreams which the patient was not able to recall the nature of the dream and the other two patients had woken up during the procedure heard some noises during the surgery and then fell asleep briefly after the episode. None of the patients reported being in pain when awake. 
Depth of Anesthesia in Off-pump CABG

Table 3: Change in mean response entropy open (RE) and in mean state entropy (SE) during surgery under anesthesia

\begin{tabular}{|c|c|c|c|c|}
\hline \multirow[b]{2}{*}{ Time } & \multicolumn{2}{|r|}{$R E$} & \multicolumn{2}{|r|}{$S E$} \\
\hline & Min-Max & Mean $\pm S D$ & Min-Max & Mean $\pm S D$ \\
\hline Pre-induction & $94-98$ & $95.33 \pm 2.31$ & $89-91$ & $90.00 \pm 1.00$ \\
\hline Immediate post-induction & $52-62$ & $58.00 \pm 5.29$ & $50-62$ & $57.33 \pm 6.43$ \\
\hline Skin incision & $58-64$ & $60.33 \pm 3.21$ & $58-62$ & $58.67 \pm 3.06$ \\
\hline Sternotomy & $54-66$ & $59.33 \pm 6.11$ & $54-64$ & $58.33 \pm 5.13$ \\
\hline LIMA & $52-60$ & $56.67 \pm 4.16$ & $54-58$ & $56.00 \pm 2.00$ \\
\hline LIMA to LAD & $56-66$ & $62.00 \pm 5.29$ & $58-68$ & $62.67 \pm 5.03$ \\
\hline RCA & $56-62$ & $58.67 \pm 3.05$ & $58-62$ & $60.67 \pm 2.31$ \\
\hline LCX & $44-66$ & $58.00 \pm 12.17$ & $42-66$ & $56.67 \pm 12.86$ \\
\hline Protamine injection & $54-61$ & $57.67 \pm 3.51$ & $56-59$ & $57.67 \pm 1.53$ \\
\hline Sternal closure & $58-64$ & $60.67 \pm 3.06$ & $56-62$ & $58.67 \pm 3.06$ \\
\hline Skin closure & $58-66$ & $60.67 \pm 4.62$ & $54-64$ & $58.00 \pm 5.29$ \\
\hline
\end{tabular}

\section{Hemodynamic Changes and Entropy Values of the Patients Aware during OPCABG Surgery}

The mean pulse rate of these patients at pre-induction was $98.67 \pm 17.01$; it increased significantly post-induction $115.33 \pm$ 5.77 , up to sternotomy. And then decreased gradually throughout the procedure. The mean systolic blood pressure of these patients at pre-induction was $143.33 \pm 6.43$, it decreased gradually and at sternotomy, it was $139.33 \pm 2.31$ and at the postoperative period, it was $118.00 \pm 6.00$. The mean diastolic pressure at pre-induction of these patients was $90.00 \pm 5.29$; it gradually decreased throughout the procedure. With a mean of $73.33 \pm 1.15$ at the postoperative period (Table 3).

The RE in these at pre-induction was $95.33 \pm 2.31$, immediate post-induction the RE was $58.00 \pm 5.29$, at various cardiac positions such as LIMA harvesting mean RE was $56.67 \pm 4.16$, during LIMA to LAD grafting it was $62.00 \pm 5.29$, at RCA grafting mean RE was $60.00 \pm 5.29$, and during LCX mean RE was $58.00 \pm 12.17$. The SE in these at pre-induction was $90.00 \pm 1.00$, immediate post-induction the SE was $57.33 \pm 6.43$, at various cardiac positions such as LIMA harvesting mean SE was $56.00 \pm 2.00$, during LIMA to LAD grafting it was $62.67 \pm 5.03$, at RCA grafting mean SE was $60.67 \pm 2.31$, and during LCX mean SE was $56.67 \pm 12.86$.

\section{Discussion}

Patients undergoing cardiovascular surgeries under general anesthesia are considered to be at an increased risk of awareness due to a combination of multiple factors like surgical, anesthetic, and patient-related. In cardiovascular surgery, most of the patients are on beta-receptor blocking drugs and other antihypertensive medication, hemodynamic variations may not be a valid guide to ascertain the depth of anesthesia.

The anesthesiologist usually assesses the depth of anesthesia based on autonomic response. Many a time, signs of lighter planes of anesthesia like a sudden shot in blood pressure and/or raised heart rate, sweating, tearing, or papillary dilatations are present. However, there are other events like hypotension, dehydration, hypoxia, hypothermia, hyperthermia, and sudden blood loss which may cause similar hemodynamic changes. Likewise, factors like demographic features of the patient, baseline tone, cardiac medications like beta-receptor-blockers, anti-hypertensive medications, inotropic medication, and vasodilators may also affect the heart rate and blood pressure.
Patient response to surgical stimulus (PRST) score, based on autonomic changes due to noxious stimulus (surgical) is not a very reliable indicator of the depth of anesthesia. ${ }^{2}$ Hemodynamic changes to surgical stimuli do not necessarily signify awareness, nor does lack of hemodynamic response warrant absence of awareness. ${ }^{2}$ In most of the cases of ASA closed claim for recall during anesthesia, there was no concomitant autonomic sign. ${ }^{1}$ Out of patients having recall during anesthesia, hypotension was seen in $15 \%$ of patients, $7 \%$ showed tachycardia, and only $2 \%$ of patients were actually moving. ${ }^{1}$

In a study by Vernon et al., it was seen that patient's response to skin incision was not predictable by hemodynamic changes. ${ }^{3}$ Till now, not much published research has been available in the Indian population regarding awareness during heart surgery. The present study was conducted to monitor the depth of anesthesia during OPCABG using an entropy monitor.

In our study, the age of all 100 patients was between 50 years and 70 years with a mean of $57.03 \pm 6.10$ years. $62.0 \%$ of cases were males and $38.0 \%$ of cases were females. The recommended range of adequate anesthesia with entropy monitoring is 40-60. During anesthesia, when SE increases above 60 , more hypnotic medication should be given. When SE is in the recommended range for adequate anesthesia but $\mathrm{RE}$ is disproportionately high, this can be interpreted as a sign of uncovered pain, and more analgesic should be administered. Several studies have suggested that entropy value is a useful parameter in improving the titration of anesthetics. It is more sensitive than the bispectral index (BIS) in detecting the deepening of anesthesia.

Several investigators have analogized spectral entropy parameters with BIS using diverse anesthetic agents during induction. Baulig et al. compared spectral entropy and BIS EEG in CABG. ${ }^{4}$ The entropy and BIS values were collated during the maintenance of anesthesia using propofol-remifentanil. The remifentanil concentration was kept constant and fentanyl was supplemented during expected painful intervals. The study showed that entropy values were comparable with the BIS, but entropy was found to be more suitable in cardiac surgery patients as it showed significantly less resistance against artifacts. The study showed a strong correlation between BIS and spectral entropy parameters.

White et al. in 2006 compared SE and BIS during induction and maintenance of anesthesia in patients undergoing major laparoscopic surgery using propofol and desflurane for anesthesia. ${ }^{5}$ 
In their study, changes in RE and SE were compared with those in the BIS during the perioperative period. The entropy module is a costequivalent alternative to the BIS monitor. In a study by Driessen et al., in cardiovascular surgery patients in whom midazolam-fentanyl total intravenous anesthesia was used, it suggested that the BIS is not a very reliable parameter of anesthetic adequacy. ${ }^{6}$

Tiren reported that only in approximately $60 \%$ of the 109 patients, entropy and BIS values are comparable during cardiopulmonary bypass (CPB) and anesthesia with midazolam/ fentanyl and propofol. ${ }^{7}$ This may be because of the predominant midazolam-fentanyl-based anesthesia with added propofol used in their study. Limited studies have been performed where only entropy is used for monitoring anesthetic depth during OPCABG.

Many anesthesiologists prefer to limit the dosage of anesthetics because of the concern of myocardial depression during OPCABG surgery. Many studies have indicated that patients anesthetized using routine clinical signs as monitoring parameters usually received more anesthesia than necessary if entropy was not monitored. The reduction of anesthetic drugs during OPCABG surgery that resulted from entropy monitoring can result in less myocardial depression, less decrease in system vascular resistance, and less need for inotropic support.

There is an increase in the prevalence of atherosclerotic disease in a patient undergoing CABG. Though there is a decline in the incidence of stroke and cognitive decline in these patients but is not uncommon. Patient-related risk factors are a degree of atherosclerosis of the aorta, carotids, and vessels of the brain. Intraoperative entropy-based monitoring could predict poor neurological outcomes after cardiac surgery in such atherosclerotic patients.

Bispectral index, a monitor for the depth of anesthesia, is an early predictor to identify patients with a good chance of recovery during therapeutic hypothermia after cardiac arrest. But SE was shown to be more effective than BIS for heart surgery during propofol-remifentanil anesthesia due to its intraoperative artifact tolerance. ${ }^{4}$

In 2012, Mohamed reported two patients with left main coronary artery disease and myocardial infarction scheduled for urgent CABG surgery. ${ }^{8}$ These patients developed abrupt decreases in RE and SE values intraoperatively. Both patients showed delayed awakening after surgery and it was correlated to non-hemorrhagic temporal-parietal cerebral infarctions.

The Modified Brice Questionnaire is an accepted tool for detecting awareness postoperatively. This open-ended questioning technique has been described by Fleisher et al. ${ }^{9}$ It is designed to elicit information in a non-alarming way and used by Pollard et al. ${ }^{10}$ The frequency of awareness reported by Pollard using this modified Brice questionnaire is $0.0068 \%$ ( 6 out of 87,361 ), which is quite less than the published frequency.

Rashad et al. in their study of 100 patients of age $>18$ years, undergoing elective CABG found a slightly higher frequency of awareness (5\%). ${ }^{11}$ Patients were interviewed $24-72$ hours after anesthesia using a modified Brice questionnaire and their anesthesia record was consulted and the drugs and doses used at induction and maintenance of anesthesia at different stages of the surgery were noted down. It was found to be less in patients who received propofol infusion during $\mathrm{CPB}$ compared to the patients who did not receive it.

In 2005, Yun et al. ${ }^{12}$ investigated the incidence of awareness in patients undergoing different kinds of cardiac surgery, the phases when awareness occurred, and the effect of CPB on the incidence of awareness in CABG in Beijing.

Post 3-6 days after surgery, 100 patients underwent CABG in Chaoyang Hospital and 100 patients who underwent CABG, and 100 patients who underwent valve replacement or septal defect repair in Fuwai hospital, Beijing were interviewed.

The frequency of awareness of patients with CABG under CPB was $4.7 \%$; in OPCABG 9.6 and $4 \%$ in septal repair or valve replacement under $C P B$. During the period of $C A B G$ under $C P B$, the incidence of awareness was not affected by $C P B$ significantly compared to patients with septal repair and valve replacement under CPB. Before bypass grafting or CPB in heart surgery, awareness occurs predominantly. Auditory perceptions were the most common form of awareness patients had. In CABG, CPB is not a significant factor that affects the incidence of awareness in patients. We interviewed to enquire about awareness, 48 hours after the surgery. A single interview has also been used by Yun et al. ${ }^{12}$ and Muralidhar et al., ${ }^{13}$ which was taken 1 week after the anesthesia and surgery. However, it has been reported that almost one-third of the patients have been identified to have awareness at follow-up interviews. ${ }^{13}$

Three patients in our study reported awareness; all three of them were posted for triple vessel grafting of the left anterior descending, left circumflex artery, and right coronary artery. Two of them were males and the other females, with a mean age of $59.33 \pm 7.02$.

The mean pulse rate of these three patients at pre-induction was $98.67 \pm 17.01$, it increased significantly post-induction $115.33 \pm$ 5.77 , up to sternotomy, and then decreased gradually throughout the procedure. The mean systolic blood pressure at pre-induction was $143.33 \pm 6.43$, it decreased gradually and at sternotomy was found to be on the higher side $139.33 \pm 2.31$ and at the postoperative period, it was $118.00 \pm 6.00$. The mean diastolic pressure at pre-induction of these patients was $90.00 \pm 5.29$; it gradually decreased throughout the procedure. With a mean of $73.33 \pm 1.15$ at the postoperative period.

The RE in them at pre-induction was $95.33 \pm 2.31$, immediate post-induction the RE was $58.00 \pm 5.29$, at various cardiac positions such as left internal mammary artery harvesting mean RE was $56.67 \pm 4.16$, during left internal mammary artery to left anterior descending artery grafting it was $62.00 \pm 5.29$, at right coronary artery grafting mean RE was $58.67 \pm 3.05$, and during left circumflex artery mean RE was $58.00 \pm 12.17$. The SE in these at pre-induction was $90.00 \pm 1.00$, immediate post-induction the SE was $57.33 \pm 6.43$, at various cardiac positions such as left internal mammary artery harvesting mean SE was $56.00 \pm 2.00$, during left internal mammary artery to left anterior descending artery grafting it was $62.67 \pm 5.03$, at right coronary artery grafting mean SE was $60.67 \pm 2.31$, and during left circumflex artery mean SE was $56.67 \pm 12.86$.

The entropy values were found to be significantly on the higher side during grafting of the left internal mammary artery to the left anterior descending artery. The values were also found to be higher than normal during left circumflex artery grafting.

We interviewed the patients 48 hours after the surgery, the last thing all of them remembered before going to sleep was being taken on the operating table, two patients remembered that they had woken up during the surgery and had heard some noises and fell asleep briefly after the episode, both of them were not in pain when they had woken up or were disturbed by it. One of them had 
vivid dreams which the patient was not able to recall the nature of the dream and was not disturbed by it.

Thus after studying 100 cases undergoing OPCABG in our institute, it has been found that the incidence of awareness was $3 \%$. Hence, it can be concluded that the incidence of awareness is high during the OPCABG. The results of this study may improve the knowledge of anesthetists and may help deal with the occurrence of intraoperative awareness.

Much larger evidence will be needed to detect whether entropy can actually be useful in decreasing intraoperative awareness in patients undergoing OPCABG surgery. ${ }^{14}$ Entropy use is extremely helpful not only in the early diagnosis of awareness during anesthesia but also to predict and diagnose intraoperative neurological complications. Thus, entropy is very useful in guiding us to modulate anesthesia techniques.

\section{References}

1. Report and findings of the 5th National Audit Project Chapter 14 AAGA in cardiothoracic .

2. Hug CC. Does opioid anaesthesia exist. Anesthesiology 1990;73: $1-4$.

3. Stump DA, Jones TJ, Rorie KD. Neurophysiologic monitoring and outcomes in cardiovascular surgery. J Cardiothorac Vasc Anesth 1999;13:600-613.

4. Baulig W, Seifert B, Schmid ER, et al. Comparison of spectral entropy and bispectral index electroencephalography in coronary artery bypass graft surgery. J Cardiothorac Vasc Anesth 2009;24: 544-549.
5. Driessen JJ, Harbers JB, Van Emond J, et al. Evaluation of the electroencephalographic bispectral index during fentanylmidazolam anaesthesia for cardiac surgery. Does it predict haemodynamicresponses during endotracheal intubation and sternotomy? Eur J Anaesthesiol 1999;16:622-627.

6. Col Ashok Sinha et al. Changes in bispectral index (BIS) values during cardiopulmonary bypass (CPB). Med J Arm Force India 2011;67(1): 38-40.

7. Tiren C. Depth of anaesthesia: a clinical study comparing entropy and BIS in cardiac surgery patients during cardiopulmonary bypass. Eur J Anaesthesiol, Euroanaesthe 2004; Abstracts Programme 2004;21(32):A-140.

8. Fleischer LH, Glass PSA. Evidence based practice of anesthesiology: can we prevent recall during anaesthesia?. Philadelphia (PA): WB Saunders; 2004. pp 223-pp 237.

9. Pollard RJ, Coyle JP, Gilber RL, et al. Intraoperative awareness in a regional medical system: a review of 3 years' data. Anesthesiology 2007;106:269-274.

10. Muralidhar K, Banakal S, Murthy K, et al. Bispectral index-guided anaesthesia for off-pump coronary artery grafting. Ann Card Anaesth 2008;11(2):105-110.

11. Rashad S, Shahab N, Safdar A, et al. Awareness during coronary artery bypass grafting surgery. Pakistan Arm Forces Med J 2011;61(4):526529.

12. Yun W, Yun Y, Yong-hai S, et al. Investigation and analysis of frequency of awareness in patients undergoing cardiac surgery in Beijing, China. CMJ 2005;118(14):1190-1194.

13. Sandin RH, Enlund G, Samuelsson P, et al. Awareness during anaesthesia: a prospective case study. Lancet 2000;355:707-711.

14. Kotur PF. Entropy - a new measure of anaesthetic depth. Indian Anaesth 2004;48(3):170-171. 